\title{
ARTICLE
}

\section{Functional cognitive disorders: identification and management}

\author{
Norman A. Poole, Sarah R. Cope, Cate Bailey \& Jeremy D. Isaacs
}

Norman Poole, MBChB, MRCPsych, MSc, MD(Res), is a consultant neuropsychiatrist at St George's Hospital in south London and editor of the BJPsych Bulletin. His special interests are functional neurological disorder, the neuropsychiatry of movement disorders and psychopathology generally. Sarah Cope, DClinPsy, MSc, BSc(Hons), is a clinical psychologist working in the Neuropsychiatry Service at St George's Hospital, London. Her research interests centre around the psychological treatment of functional neurological disorder. Cate Bailey, MBBS, MRCPsych, MRes, is a specialist registrar in general adult and old age psychiatry and a former academic clinical fellow in old age psychiatry at Queen Mary University, London. She is an honorary clinical research fellow at Queen Mary University of London. Her previous research has focused on communication of dementia diagnoses and assessment of functional memory disorders. Jeremy Isaacs, MA,

MBBS, PhD, MRCP, is a consultant neurologist at St George's Hospital, London, and Kingston Hospital,

Kingston upon Thames, and an honorary senior lecturer at St George's University of London. His current research interests are in functional cognitive disorders, delirium and clinical trials in Alzheimer's disease and vascular dementia.

Correspondence Dr Norman

A. Poole, Department of

Neuropsychiatry, South West London \& St George's Mental Health

NHS Trust, St George's Hospital, Blackshaw Road, London

SW17 00T, UK.

Email: Norman.poole@swlstg.nhs.uk

First received 26 Feb 2019

Final revision 17 Apr 2019

Accepted 29 Apr 2019

\section{Copyright and usage}

(c) The Royal College of Psychiatrists 2019

A podcast is available to accompany this article at: soundcloud.com/ bjpsych/bja-2019-38

\section{SUMMARY}

We review the various functional cognitive disorders (FCDs) - complaints about memory function or another cognitive process in the absence of relevant neuropathology. These are increasingly coming to the attention of psychiatrists and neurologists and FCD encompasses some newly recognised conditions in addition to classic types such as pseudodementia and psychogenic amnesia. The clinical features, neuropsychological findings and treatment are presented and discussed.

\section{LEARNING OBJECTIVES}

After reading this article you will be able to:

- describe clinical features of FCD and how it differs from neurodegenerative causes of cognitive impairment

- be able to subclassify cases of FCD using the proposed nosology

- understand how to discuss the diagnosis with the patient and explain how the symptoms arise.

\section{DECLARATION OF INTEREST}

None.

\section{KEYWORDS}

Clinical neurology; cognitive neuroscience; dissociative disorders; functional cognitive disorders.

Functional cognitive disorder (FCD) denotes a complaint about memory function or, less commonly, another cognitive process in the absence of relevant neuropathology and with evidence of inconsistency between symptoms reported and signs identified at assessment. The prototypical FCDs are dissociative fugue and amnesia, but these account for only a tiny proportion of people presenting with subjective cognitive complaints. The relationship between such subjective complaints and current or future dementia is complex. A meta-analysis found that older people (with a mean age of 71.6 years) with subjective memory complaints are twice as likely to develop dementia as those without SMCs over an average follow-up period of nearly 5 years (Mitchell 2014). However, a more recent 6-year follow-up study of subjective memory complaints in 81 younger patients (the mean age was 61 years) found that $86 \%$ were stable or improved (Hessen 2017).
Other follow-up studies have demonstrated similar rates of stability, with low rates of progression to neurodegenerative disorders (Vestberg 2010). In a large study of cognitively well older people, it was reduced awareness of memory impairment that predicted near-term onset of dementia (Wilson et al 2015). Indeed, there is only a small association between subjective report and objective cognitive performance, and it is weaker than the link between subjective report and affect (Burmester 2016). Given that other functional neurological disorders can be comorbid with an underlying neurodegenerative condition - so-called functional overlay it would not be surprising for an FCD to occur in the context of prodromal dementia or, increasingly, for it to be triggered by the identification of dementia biomarkers such as being positive for APOE. In the absence of relevant evidence, this remains a conjecture.

With the expansion of diagnostic memory clinics over the past decade, increasing numbers of people with FCD are being identified, which reflects greater numbers of patients attending without neurodegenerative conditions (Larner 2014). According to studies in the UK and the USA, between 33 and $56 \%$ of persons assessed in memory clinics are diagnosed as 'normal' or 'no cognitive disorder' (Pennington 2015). It is as yet unclear how to conceptualise and manage this group of patients. Most are discharged back to primary care without a diagnosis or are given the placeholder label of mild cognitive impairment (MCI), which, we should emphasise, is not a synonym for FCD. Although evidence-based interventions for the disorder are lacking, there are some findings to suggest that strategies that focus on expectations, cognitive restructuring or psychoeducation may be helpful (Metternich 2010; Bhome 2018). However, such interventions are generally not available in diagnostic memory clinics: $73 \%$ of memory service psychiatrists surveyed in the UK in 2015 discharged patients with functional disorders of memory to primary care and among those who did offer treatment for FCD there was no agreement on what was appropriate (Bailey 2017). Here, we provide an up to date summary of research into the nosology, diagnostic criteria, aetiology, causal mechanisms and management of this emerging clinical conundrum. 


\section{Nosology}

Before discussing a proposed nosology for FCD it is worthwhile noting the multitude of terms used by cognitive neurologists and psychiatrists to describe the condition. Examples of these are listed in Box 1. Some terms seem to minimise and normalise the state, whereas others posit an underlying cause. The condition is not well captured in ICD-10 (World Health Organization 1992), which largely emphasises dissociative fugue and amnesia to the exclusion of other forms of the disorder. DSM-5 (American Psychiatric Association 2013) distinguishes between dissociative amnesia and other functional neurological disorders, which would include some of the categories described below. DSM-5 has now removed the need for a prior trauma or stressor from the diagnostic criteria, given that this is not always found and emphasises inconsistency between presentation and signs elicited on examination.

Given this lack of order, Stone et al (2015) proposed a nosology of FCD. They described six overlapping categories:

1 memory symptoms in the context of anxiety/ depression

2 normal memory lapses that become a focus of concern to the patient

3 isolated functional memory complaints that go beyond normal lapses but cannot be accounted for by anxiety/depression

4 hypochondriacal disorder with dementia as the focus

5 memory symptoms in the context of another functional disorder

6 retrograde dissociative 'psychogenic' amnesia.

\section{B0X 1 Terminology}

Terms used in the clinic and in research to describe complaints about memory function or another cognitive process in the absence of relevant neuropathology include:

- functional cognitive disorder (FCD)

- cognitive impairment secondary to complex psychological processes

- attentional amnestic disorder

- subjective memory impairment

- hypochondriasis

- worried well

- age-related forgetfulness

- functional memory symptoms (Stone 2015)

- functional memory disorder (Schmidtke 2008)

- cogniform disorder (Delis 2007)

(Bailey et al 2017)
An addition to this list might be the recently described behavioural-variant frontotemporal dementia (bvFTD) phenocopy syndrome. This syndrome presents with the clinical symptoms and signs of bvFTD but structural and functional imaging are normal (Kipps 2009) and there is an absence of progression, even up to 21 years after diagnosis (Devenney 2018). While it is increasingly accepted that this is not a neurodegenerative condition, it is unclear exactly where this disorder sits in our nosology and it likely arises through a mechanism distinct from the functional cognitive disorders.

\section{Category 1: memory symptoms in the context of anxiety/depression}

Memory symptoms and difficulty with concentration are commonly reported in anxiety and depression and are part of the diagnostic criteria. There is a recall bias such that positive memories are less readily accessed, and subtle deficits of attention and executive functioning are found on neuropsychological testing: impaired set-shifting and verbal fluency are the most common findings (Rock 2014). A systematic review conducted by Hasselbalch et al (2011) demonstrated that the executive and attentional dysfunction persists after remission of the depressed mood, leading the review authors to argue that cognitive impairment is an intrinsic component of depression and not merely an epiphenomenon. Indeed, cognitive dysfunction may adversely affect occupational performance more than the depressed mood (McIntyre 2015). Drug manufacturers have identified this too and recent studies of the antidepressant vortioxetine suggest that the drug significantly improves cognitive functioning across a range of domains compared with placebo and citalopram, independent of its impact on mood (McIntyre 2016).

\section{Pseudodementias}

On occasion, memory symptoms dominate the picture and present as pseudodementia, a term first employed by Wernicke to describe 'a chronic hysterical state mimicking mental weakness' (quoted in Berrios 1996: p. 190). Depressive, manic and anxious-type pseudodementias have been described. The depressive form is the most common; indeed, it is almost synonymous with the term pseudodementia. It is usually distinguished from dementia by a precisely dated onset with rapid deterioration and reduced effort on bedside and formal testing of cognition: the classic 'I don't know' responses described by Wells (1979). Lishman (1978: p. 572) described such patients in the following terms: '[they are] slow to grasp essentials, thinking is laboured and behaviour becomes 
generally slipshod and inefficient. Events fail to register, either through lack of ability to attend and concentrate or on account of [...] inner preoccupations'. The concept of depressive pseudodementia has been criticised on the grounds that it is poorly defined and late-onset depression often heralds a subsequent neurodegenerative dementia, so may not be pseudo at all (Saez-Fonseca 2007). It certainly carries a poor prognosis and high mortality rate. Nevertheless, the 'pseudo' component captures the potential for recovery and absence of neurodegeneration (Sachdev 2003) more succinctly than modern terms such as depression-executive dysfunction syndrome. These matters are beyond the scope of this article, but the point to bear in mind is that depressive pseudodementia is an at least partially reversible cause of cognitive impairment, so should be identified and treated in its own right, whatever the future holds.

Patients with manic pseudodementia are distractible to the point that they cannot even follow their own train of thought. Severe inattention masquerades as delirium, producing disorientation, disorganised thinking, hallucinations, delusions and dysphoria suggestive of gross cognitive impairment. Occasionally, obsessive ruminations are so severe the sufferer cannot attend to anything else, leading to self-neglect and apparent memory loss. Usually in both manic and obsessive cases, the patient's psychiatric history and a thorough mental state examination should alert the clinician to the presence of associated symptoms and signs. However, it should be noted that bipolar disorder can be both a risk factor for, and a mimic of, frontotemporal dementia (Gambogi 2018).

Malingering and factitious disorder are potential mimics of pseudodementia, particularly in the medico-legal setting, but will not be discussed further here. Dissociative amnesia has sometimes been classified as a non-depressive pseudodementia, but Stone et al (2015) include it as a separate category: retrograde dissociative 'psychogenic' amnesia (see below).

\section{Category 2: normal memory lapses that become a focus of concern}

Memory lapses are entirely normal phenomena and occur surprisingly frequently in healthy control populations. For instance, clinicians managing FCD often show patients normative data on forgetfulness to highlight the frequency and severity of such lapses. One of us (N.P.) cites evidence about so-called flashbulb memories, episodes so dramatic they are apparently seared in the mind. Even these are prone to significant errors and frank misremembering. Psychologists studied these at the time of the space shuttle Challenger explosion by interviewing study participants within $24 \mathrm{~h}$ of the disaster. In $25 \%$ of participants not one detail of the original experience was remembered correctly 2.5 years later. On average, fewer than half the original details were reported accurately at this follow-up interview (Neisser 1992). The increased attention on dementia from politicians and the media likely makes a number of people overly concerned about normal lapses, especially where direct experience of dementia, its frightening implications for an increasingly valued autonomy and the inexorable rise of the knowledge economy make these particularly salient.

\section{Category 3: isolated memory complaints without anxiety/depression}

Isolated memory complaints are probably the most common presentation to be referred to secondary care. They present a challenge because the lack of associated features requires a new explanatory model that enables practitioners to explain how the symptoms arise that does not involve a causal role for mood and health anxiety. These patients complain of significant problems that have an impact on their social and occupational functioning but are incongruent with objective testing, although some impairment may be found. The degree of impairment required for a diagnosis of FCD is as yet unsettled. Some researchers insist that no impairment on formal neuropsychological testing should be evident, whereas others allow a decline of less than 1.5 s.d. from expected functioning (Schmidtke 2008). A mild degree of anxiety and/ or depression is frequently reported but this does not meet the threshold for a formal diagnosis of affective disorder, is inadequate as an explanation for the severity of symptoms reported and patients are not overtly hypochondriacal.

Berrios et al (2000) identified two clinically recognisable subgroups from 49 patients who attended a memory clinic in the UK. They found no biomedical or neuropsychological abnormality, nor any ICD-10 psychiatric diagnosis. Twenty-two of the patients were classed as having 'functional cognitive disorganisation'. Mainly women, these individuals typically had low educational attainment, a pattern of short-lived relationships and described a life event triggering their memory complaint. They had a stronger tendency for dissociation and less anxiety than the remaining 27 patients, who were predominantly younger, bright, well-educated, perfectionistic men who displayed 'covert, over-controlled anxiety'. This second group complained of impaired autobiographical and semantic memory and the authors classed them as suffering 'mnestic hypochondria'. However, they were not diagnosed with hypochondriacal disorder, as that diagnosis involves 
misinterpretation of, and preoccupation with, somatic symptoms only. In our nosology, Berrios et al's mnestic hypochondria cases probably fall into the next category.

\section{Category 4: hypochondriacal disorder with dementia as the focus}

An established hypochondriacal disorder focused on dementia is discussed infrequently in the literature and is uncommon in clinical practice. However, the fear of developing dementia is highly prevalent among people in middle and early old age (Kessler 2012). Whereas patients with a different primary anxiety disorder and cognitive symptoms tend not to dwell excessively on a specific diagnosis, the thoughts of these hypochondriacal patients (socalled 'dementia worry') are dominated by the prospect of dementia, and each memory lapse only serves to confirm their fears. As in other hypochondriacal disorders, individuals often spend an excessive amount of time reading about the dreaded condition, request expensive, even invasive, investigations and seek reassurance from carers and professionals, which gives only transient relief but positively reinforces the reassurance-seeking behaviour.

\section{Category 5: memory symptoms in the context of another functional disorder}

Impaired memory and concentration with wordfinding difficulties are frequent complaints in all functional disorders (Teodoro 2018). They are a core feature in chronic fatigue syndrome and fibromyalgia (Wessely 1999) and occur in around 50\% of those with functional movement disorder (Heintz 2013). There is a striking similarity in the descriptions given by those with pure FCD and in these other functional groups, leading some to argue for a common causal mechanism (see below).

\section{Category 6: retrograde dissociative 'psychogenic' amnesia}

Psychogenic amnesia is the sudden loss of autobiographical memories, presumed to be a response to or defence against an overwhelming psychological stressor. It is such a striking and dramatic phenomenon that it captures the imagination of clinicians and novelists alike, dominating, perhaps unhelpfully, theoretical thinking on FCD generally. Harrison et al (2017) recently proposed three distinct subtypes based on 53 patients presenting to St Thomas' Hospital, London, between 1990 and 2008, representing the largest case series to date of this rare condition. These subtypes, described in Box 2, are fugue state, focal retrograde amnesia and memory gaps. Interestingly, the patients
BOX 2 Subtypes of psychogenic amnesia

Fugue state: sudden and complete autobiographical amnesia and loss of personal identity, with or without a period of wandering. Fugues tend to last a few days only but residual amnesia for the period of the fugue often persists.

Focal retrograde amnesia: following minor head injury or neurological event there develops a sudden and prolonged amnesia for autobiographical memories with a reversed temporal gradient, i.e. recall for early life is worse than for recent events.

Memory gaps: discrete 'islands' of amnesia for autobiographical memory associated with a specific psychosocial stressor or trauma, which can last from hours to weeks. Some report multiple amnesic gaps.

(Harrison et al 2017)

ranged in age from early 20s to mid-60s and threequarters of them were men. Their psychogenic amnesia was often preceded by a major stressor in conjunction with a history of depression and a recent or previous minor neurological event, such as mild head injury or migraine. A significant number of patients transitioned from fugue into focal retrograde amnesia.

\section{Assessment}

Patients presenting with complaints about memory function require standard psychiatric and neurological history and examination, as for any patient presenting with cognitive symptoms. We are here only concerned with signs and symptoms that point to FCD. It should be emphasised that these conditions are not diagnoses of exclusion but have positive symptoms and signs that should become well-known to clinicians working in memory clinics, liaison psychiatry, neurology and neuropsychiatric settings. The issue of special investigations will be addressed below.

Typical complaints described by those with FCD include forgetting an intended action while on the way to carry it out, inability to recall well-founded memories (such as PIN numbers), disruptions in the flow of thoughts and conversations, spoonerisms, and forgetting future tasks and appointments (Schmidtke 2008). Others have described tip-ofthe-tongue phenomena: the inability to retrieve a word or fact, with subjective awareness that it is 'nearly there'. Patients often report having forgotten names of colleagues, where they parked their car, what occurred during a journey, or an apparently important piece of autobiography, such as a recent holiday or significant family event. These can interfere with usual activities of daily living and 
performance at work. There is usually a discrepancy between the severity of the complaints and ability to function in day-to-day life, however.

A hallmark of all functional disorders is the presence of internal and external inconsistencies and incongruities, and these should be elicited in patients complaining of cognitive symptoms in exactly the same way as for a functional motor or seizure disorder. In the context of FCD, an internal incongruity would be the ability to describe an episode of memory loss in great detail, cognitive symptoms that come and go according the patient's circumstances, and a high burden of subjective cognitive difficulties that have not been noticed by family or co-workers. External incongruities include objectively normal performance on cognitive tests that would be reliably abnormal in people with dementia. Arguably, this is less reliable than the internal incongruities described above because, although normal performance on standard neuropsychometric instruments has been shown for FCDs at the group level, at the individual level people with FCD can underperform on cognitive testing for a variety of reasons.

A number of clinical signs have been described that point towards the diagnosis of functional cognitive disorder in memory clinic settings (Bailey 2018), and these are outlined in Box 3. Reuber et al (2018) operationalised these features into a 'diagnostic scoring aid', which in a pilot study was able to distinguish patients with neurodegenerative dementias from those with FCDs with a sensitivity of $86.7 \%$ and a specificity of $100 \%$.

These signs and symptoms are most typical of categories 1-5 above. The memory complaints

BOX 3 Clinical signs suggesting a diagnosis of functional cognitive disorder

The following clinical signs point towards the diagnosis of functional cognitive disorder in memory clinic settings:

- attending alone

- the ability to provide detailed answers about previous memory failures and personal history

- responding appropriately to compound questions

- answering quickly and loquaciously

- not turning the head to a companion (if present) when providing details

- the patient being more worried than others about the problem

- referring back to earlier answers given over the course of the interview ('as I said before').

- 'memory perfectionism': frustration with lapses that others would consider relatively trivial

(Pennington et al 2015; Bailey et al 2018) described by those with psychogenic amnesia are more pervasive and profound, while those with pseudodementia may not spontaneously complain of any cognitive difficulty at all but, because of apathy and amotivation, respond to questions by saying 'I don't know' (Wells 1979). To ensure that an atypical presentation of Alzheimer's disease, which occurs more commonly in younger-onset patients, is not being missed (Koedam 2010) the neurological examination should include tests for apraxia, such as mimicking use of a tool and copying meaningless hand gestures.

\section{Neuropsychological assessment}

There is no specific pattern of impairment on neuropsychological testing, and underperformance may be attributable to over-anxiety or apathy observed by the assessor. Wakefield and colleagues compared neuropsychological test performance of healthy controls with that of people with FCD or amnestic MCI (Wakefield 2018). Although those with FCD scored normally on memory tests, their performance on letter fluency and digit cancellation tasks was not significantly different from those with amnestic MCI. The implications of this finding are unclear, but these tasks might be more susceptible to subtle decrements in attention in FCD that persist even in the calm testing environment.

Teodoro et al (2018) recently performed a systematic review of cognitive symptoms and neuropsychological findings in fibromyalgia, chronic fatigue syndrome and non-cognitive functional neurological disorders, a group corresponding to FCD category 5 (memory symptoms in the context of another functional disorder) in the classification shown above. Importantly, the published literature does not show consistent deficits on tests of memory and other domains typically implicated in neurodegenerative dementias. However, there was a tendency for people with these conditions to show poor selective and divided attention, with slowed processing speed (Teodoro 2018). The authors proposed that functional memory complaints generally are underpinned by a lack of attentional reserve, which could increase susceptibility to distraction and slow down information processing. This, combined with memory perfectionism, the overinterpretation of memory lapses and heightened self-monitoring for cognitive errors, might disproportionately disrupt real-life multi-tasking while being difficult to assess on standard neuropsychological testing. Of note, they also identified that only a small proportion of patients failed validity testing, so these tests must be interpreted with extreme caution and are an unreliable guide to diagnosis. 
Harrison et al (2017) showed that psychogenic functional amnesia is associated with impaired verbal fluency and a flat or reverse temporal gradient (a flat gradient occurring only in fugue states) for personal semantic facts and episodic memories that at least partially recovers over time. However, they found no difference between individuals with psychogenic amnesia and both normal and neurological controls on anterograde memory tests. Nevertheless, the psychogenic amnesia group did show improved scores on those tests after the acute phase of the episode ended. The most important features pointing to psychogenic rather than neurological amnesia were: loss of personal identity; the presence of depression; inability to recognise family; interpersonal difficulties; financial or employment problems; previous diagnosis of posttraumatic stress disorder; and, interestingly, a history of head injury. Kopelman (2000) has argued that a major psychosocial stressor combined with depressed mood and previous experience of a brief amnesia, such as occurs in minor head injury, could cause 'frontal' inhibition of autobiographical memory retrieval processes.

\section{Biomedical investigations}

The extent to which the symptoms of FCD overlap with those of organic disorders is unknown. Most organic differentials, for example endocrine disorders and sleep apnoea, can be excluded by careful history-taking and targeted investigations such as blood tests and polysomnography. There are no imaging or biomarker tests that can confirm a diagnosis of FCD. The utility of these tests is in ruling out organic mimics, notwithstanding the inherent limitations of structural imaging in confirming the common dementia subtypes. Although some authors stress the importance of performing 'rule out' tests (Pennington 2015), in our opinion there is no requirement to perform cranial imaging where a diagnosis of FCD can be made confidently on clinical grounds.

\section{Mechanism}

The precise mechanism for the impairments described in FCD is unknown. However, Teodoro et al (2018) recently proposed a causal mechanism for cognitive symptoms in all functional somatic syndromes (e.g. chronic fatigue syndrome, fibromyalgia, functional neurological disorder) that is likely to be applicable to FCD generally, although the details may differ for memory and concentration difficulties linked to anxiety/depression and the pseudodementias. As poor selective and divided attention and slowed information processing are described in all functional cognitive syndromes, a vulnerability to distraction might impair memory registration, leading to difficulty with later recall. They argue that memory perfectionism, with an excessive self-monitoring for cognitive errors and memory lapses (indeed, an excessive attention directed towards somatic symptoms of all types), is compounded by catastrophic thinking that then drives reassurance- and help-seeking.

Teodoro and colleagues propose that a key step in the development of FCD is a switch from an automatic (or implicit) to a controlled (or explicit) mode of cognitive processing (Teodoro 2018). This hypothesis has previously been elucidated with respect to functional movement disorders. Controlled processes are significantly less efficient and associated with a greater degree of conscious effort. Such a mechanism might explain why people with chronic fatigue syndrome report a heightened perception of effort when undertaking neuropsychological assessment. A heightened perception of cognitive effort may also contribute to the experience of illness, because of the extreme effort invested in routine daily tasks.

\section{Treatment}

\section{Explanation and reassurance}

As with all functional neurological disorders, a mainstay of treatment is to carefully explain to the patients what they have, what has been excluded and how you arrived at the diagnosis (Stone 2011). It is useful to focus on the mechanism by which symptoms arise and discuss any remediable maintaining factors. It is important to take the patient's symptoms and suffering seriously and to assure them that they are believed, but also to emphasise that the condition is common, reversible and can be managed through self-help strategies. Although it is useful to discuss the role of mood and anxiety, make sure not to 'explain away' all the cognitive symptoms as 'just' depression, because it is likely that the patient will have heard this many times before from other clinicians. Expect resistance to such a suggestion. However, if the person is taken seriously, they may in time acknowledge greater depression and anxiety than admitted initially. Neuroimaging and neuropsychometric results need to be discussed, which can complicate matters when an incidental finding is discovered, or neuropsychology reveals blanket impairments and failed validity testing.

\section{Memory training and expectancy modification/ change}

Specific psychological treatment of FCD has usually been separated into two strands: memory training and expectancy modification. Memory-training 
MCO answers

$1 d \quad 2 c \quad 3$ e $4 d \quad 5 b$ strategies include such techniques as 'the method of loci' (e.g. memory palaces and such-like). Metternich et al (2010) reviewed 14 non-pharmacological interventions for people with FCD lacking objective impairment on neuropsychological tests and found that the only intervention to improve participants' subjective experience of impaired memory was, in their terms, 'expectancy change'. One of the interventions reviewed was their pilot randomised controlled trial of 13 sessions of group cognitivebehavioural therapy (CBT) intended to encourage such change (Metternich 2008). This found that patients in the treatment group had significantly increased memory-related self-efficacy at the end of the intervention and at 6-month follow-up.

'Expectancy change' interventions aim to alter patients' beliefs and attitudes about their own memory through the use of various combinations of cognitive restructuring, psychoeducation about normal memory processes and ageing and the relationships between memory, stress and mood, and the teaching of stress-management techniques. Expectancy change forms the basis of a brief psychological group intervention run by two of us (S.C. and N.P.). In the early stages, we counter memory perfectionism (Pennington 2015) by discussing normal memory lapses and rates of forgetting found in young healthy people and also highlight the role of 'secondary suffering' - the loss of valued activities and roles owing to preoccupation with the problem. Using a cognitive-behavioural framework, strongly influenced by ideas from acceptance and commitment therapy (a type of CBT), we aim to increase individuals' awareness of the relationships between their memory lapses, their thoughts and beliefs about their memory, their feelings and their behavioural responses. It posits that the problem is not only that they experience memory lapses ('primary suffering'), but also that their thoughts, behaviours and feelings in response to these lapses can create 'secondary suffering' in their lives.

\section{Medication}

Psychopharmacology has a very limited role in treating FCD. If significant depression and/or anxiety is found, it should be treated appropriately. Given the alleged potential benefits of vortioxetine for cognitive symptoms in depressive disorders (McIntyre 2016), N.P. has trialled its use in patients with FCD and comorbid depression but without any dramatic successes.

\section{Abreaction for dissociative amnesia}

Abreaction, which involves interviewing individuals under the influence of a drug, has a place in psychiatric folklore as particularly beneficial for dissociative amnesias, and a systematic review (Poole 2010 ) found that $88 \%$ of treated patients did improve. However, it is likely this figure is heavily influenced by publication bias and abreaction is now rarely, if ever, attempted with such patients. Most people with dissociative amnesia will, in time, recover spontaneously (Harrison 2017). They should probably be followed up by psychiatric services until they do, because significant psychological and social stressors are likely to come to light over time, so premature discharge may lead general practitioners to refer unnecessarily for further investigations; also, mental health professionals can usefully guide the patient back towards independence.

\section{Conclusions}

Functional cognitive disorder, which is characterised by absence of neuropathology in the presence of inconsistency between complaints about memory problems and signs elicited on examination, is a common condition that psychiatrists will increasingly encounter as populations age and employment becomes ever more cognitively demanding. It remains poorly classified in diagnostic manuals, but the variety of functional cognitive disorders is reasonably well understood by clinicians. Nevertheless, we remain uncertain about prognosis, and treatments, both psychological and pharmacological, need to be developed and studied to reduce the burden on patients and healthcare systems.

\section{References}

American Psychiatric Association (2013) Diagnostic and Statistical Manual of Mental Disorders (5th edn) (DSM-5). American Psychiatric Publishing.

Bailey C, Bell SM, Blackburn DM (2017) How the UK describes functional memory symptoms. Psychogeriatrics, 17: 336-7.

Bailey C, Poole NA, Blackburn DJ (2018) Identifying patterns of communication in patients attending memory clinics: a systematic review of observations and signs with potential diagnostic utility. British Journal of General Practice, 68: e123-38.

Berrios GE (1996) The History of Mental Symptoms: Descriptive Psychopathology since the Nineteenth Century. Cambridge University Press.

Berrios GE, Marková IS, Girala N, et al (2000) Functional memory complaints: hypochondria and disorganization. In Memory Disorders in Psychiatric Practice (eds GE Berrios, JR Hodges): 384-99. Cambridge University Press.

Bhome R, Berry AJ, Huntley JD, et al (2018) Interventions for subjective cognitive decline: systematic review and meta-analysis. BMJ Open, 8 : e021610.

Burmester B, Leathem J, Merrick P (2016) Subjective cognitive complaints and objective cognitive function in aging: a systematic review and meta-analysis of recent cross-sectional findings. Neuropsychology Review, 26: 376-93

Delis DC, Wetter SR (2007) Cogniform disorder and cogniform condition: proposed diagnoses for excessive cognitive symptoms. Archives of Clinical Neuropsychology, 22: 589-604. 
Devenney E, Swinn T, Mioshi E, et al (2018) The behavioural variant frontotemporal dementia phenocopy syndrome is a distinct entity - evidence from a longitudinal study. BMC Neurology, 18(1): 56.

Gambogi LB, Guimarães HC, de Souza LC, et al (2018) Long-term severe mental disorders preceding behavioral variant frontotemporal dementia: frequency and clinical correlates in an outpatient sample. Journal of Alzheimer's Disease, 66: 1577-85.

Harrison NA, Johnston K, Corno F, et al (2017) Psychogenic amnesia: syndromes, outcome, and patterns of retrograde amnesia. Brain, 140: 2498-510.

Hasselbalch BJ, Knorr U, Kessing LV (2011) Cognitive impairment in the remitted state of unipolar depressive disorder: a systematic review. Journal of Affective Disorders, 134: 20-31.

Heintz CE, van Tricht MJ, van der Salm SM, et al (2013) Neuropsychological profile of psychogenic jerky movement disorders: importance of evaluating non-credible cognitive performance and psychopathology. Journal of Neurology, Neurosurgery \& Psychiatry, 84: 862-7.

Hessen E, Eckerstrom M, Nordlund A, et al (2017) Subjective cognitive impairment is a predominantly benign condition in memory clinic patients followed for 6 years: the Gothenburg-0slo MCI Study. Dementia and Geriatric Cognitive Disorders Extra, 7: 1-14.

Kessler E-M, Bowen CE, Baer M, et al (2012) Dementia worry: a psychological examination of an unexplored phenomenon. European Journal of Ageing, 9: 275-84.

Kipps CM, Hodges JR, Fryer TD, et al (2009) Combined magnetic resonance imaging and positron emission tomography brain imaging in behavioural variant frontotemporal degeneration: refining the clinical phenotype. Brain, 132: 2566-78

Koedam EL, Lauffer V, van der Vlies AE, et al (2010) Early- versus lateonset Alzheimer's disease: more than age alone. Journal of Alzheimer's Disease, 19: 1401-8.

Kopelman MD (2000) Focal retrograde amnesia and the attribution of causality: an exceptionally critical review. Cognitive Neuropsychology, 17: 585-621.

Larner AJ (2014) Impact of the National Dementia Strategy in a neurologyled memory clinic: 5-year data. Clinical Medicine, 14: 216

Lishman WA (1978) Organic Psychiatry: The Psychological Consequences of Cerebral Disorder. Blackwell Scientific Publications.

McIntyre RS, Soczynska JZ, Woldeyohannes HO, et al (2015) The impact of cognitive impairment on perceived workforce performance: results from the International Mood Disorders Collaborative Project. Comprehensive Psychiatry, 56: 279-82.

McIntyre R, Harrison J, Loft H, et al (2016) The effects of vortioxetine on cognitive function in patients with major depressive disorder: a meta-anaIysis of three randomized controlled trials. International Journal of Neuropsychopharmacology, Jun 15: doi 10.1093/ijnp/pyw055 [Epub ahead of print]

Metternich B, Schmidtke K, Dykierek P, et al (2008) A pilot group therapy for functional memory disorder. Psychotherapy and Psychosomatics, 77. 259-60.

Metternich B, Kosch D, Kriston L, et al (2010) The effects of nonpharmacological interventions on subjective memory complaints: a systematic review and meta-analysis. Psychotherapy and Psychosomatics, 79: 6-19.
Mitchell AJ, Beaumont H, Ferguson D, et al (2014) Risk of dementia and mild cognitive impairment in older people with subjective memory complaints: meta-analysis. Acta Psychiatrica Scandinavica, 130: 439-51.

Neisser U, Harsh N (1992) Phantom flashbulbs: false recollection of hearing the news about Challenger. In Affect and Accuracy in Recall: Studies of "Flashbulb" Memories (eds E Winograd, U Neisser): 9-31. Cambridge University Press.

Pennington C, Hayre A, Newson M, et al (2015) Functional cognitive disorder: a common cause of subjective cognitive symptoms. Journal of Alzheimer's Disease, 48(suppl 1): S19-24.

Reuber M, Blackburn DJ, Elsey C, et al (2018) An interactional profile to assist the differential diagnosis of neurodegenerative and functional memory disorders. Alzheimer Disease \& Associated Disorders, 32 : 197-206.

Rock PL, Roiser JP, Riedel WJ, et al (2014) Cognitive impairment in depression: a systematic review and meta-analysis. Psychological Medicine, 44: 2029-40.

Sachdev P, Reutens S (2003) The nondepressive pseudodementias. In Dementia: Presentations, Differential Diagnosis, and Nosology (eds VOB Emery, TE Oxman): 418. JHU Press.

Saez-Fonseca JA, Lee L, Walker Z (2007) Long-term outcome of depressive pseudodementia in the elderly. Journal of Affective Disorders, 101: $123-9$.

Schmidtke K, Pohlmann S, Metternich B (2008) The syndrome of functional memory disorder: definition, etiology, and natural course. American Journal of Geriatric Psychiatry, 16: 981-8.

Stone J, Carson A (2011) Functional neurologic symptoms: assessment and management. Neurologic Clinics, 29: 1-18.

Stone J, Pal S, Blackburn D, et al (2015) Functional (psychogenic) cognitive disorders: a perspective from the neurology clinic. Journal of Alzheimer's Disease, 49(suppl 1): S5-17.

Teodoro T, Edwards MJ, Isaacs JD (2018) A unifying theory for cognitive abnormalities in functional neurological disorders, fibromyalgia and chronic fatigue syndrome: systematic review. Journal of Neurology, Neurosurgery, and Psychiatry, 89: 1308-19.

Vestberg S, Passant U, Elfgren C (2010) Stability in the clinical characteristics of patients with memory complaints. Archives of Gerontology and Geriatrics, 50: e26-30

Wakefield SJ, Blackburn DJ, Harkness K, et al (2018) Distinctive neuropsychological profiles differentiate patients with functional memory disorder from patients with amnestic-mild cognitive impairment. Acta Neuropsychiatrica, 30: 90-6.

Wells CE (1979) Pseudodementia. American Journal of Psychiatry, 136: 895-900.

Wessely S, Nimnuan C, Sharpe M (1999) Functional somatic syndromes: one or many? Lancet, 354 : 936-9.

Wilson RS, Boyle PA, Yu L, et al (2015) Temporal course and pathologic basis of unawareness of memory loss in dementia. Neurology, 85: 984-91.

World Health Organization (1992) The ICD-10 Classification of Mental and Behavioural Disorders: Clinical Descriptions and Diagnostic Guidelines. WHO 


\section{MCOs}

Select the single best option for each question stem

1 Functional cognitive disorder (FCD) is:

a another name for mild cognitive impairment (MCl)

b a form of malingering

c always caused by depression and/or anxiety

$\mathrm{d}$ a common presentation at memory clinics

e an early sign of dementia.

2 Psychogenic amnesia is:

a the most common form of FCD

b always preceded by a fugue state

c more common in males than females

d caused by minor head injury

e identified by having a clear temporal gradient for autobiographical memory.
3 Patients with FCD:

a generally bring their partner/relative/friend to clinic appointments

b look to their partner/relative/friend to answer questions for them

c need cranial CT or MRI scans to exclude a neurological diagnosis

d just require reassurance

e can be reliably identified by how they answer questions about their memory/cognitive functioning.

4 For patients presenting with suspected FCD, neuropsychological assessment:

$a$ is essential in all cases

b always shows no abnormality c reveals a distinctive pattern of impairment

$\mathrm{d}$ often demonstrates poor selective and divided attention

e should be ignored when discussing diagnosis with the patient.

5 Treatment of FCD:

a starts with an antidepressant

b aims to modify expectations and memory perfectionism

c teaches patients memory-training strategies

$d$ by means of group CBT focuses on the primary suffering of memory failure

$\mathrm{e}$ is best left to specialists. 